\title{
Institutionalizing Operations Analysis for Security and Defense in Bulgaria
}

\author{
Klaus Niemeyer, Velizar Shalamanov, and Todor Tagarev *
}

During the period of preparation for membership and accession to NATO, the administration of the security sector in Bulgaria had very limited access to expertise and tools to support decision making on key functions for the effective management of defense, such as long-term force planning, operations planning, and acquisition management. The institutes that had been responsible for such tasks were closed down as part of the downsizing of the defense establishment in the 1990s. A small number of researchers and analysts were transferred to other defense organizations, primarily to the Defense Advanced Research Institute (DARI), which is part of the "G.S. Rakovski" Defense and Staff College (DSC) in Sofia. Institutes of the Bulgarian Academy of Sciences (BAS) employed others. The Operations Research (OR) Department in the Institute of Mathematics and Informatics in the BAS was primarily oriented toward theoretical studies and teaching. Some of the legacy software tools for decision support and Computer Assisted Exercises (CAX) packages were also available in DARI, but the level of connection between this institute and the larger civilian operations research community was limited.

This was all that allowed Bulgaria to preserve a limited capacity to support defense decision making, but there were no opportunities to develop new methodologies and tools to address the new challenges to security and the needs of other security sector organizations. Given the lack of infrastructure and the limited access to security organizations, the small Bulgarian operations analysis (OA) community has not been utilized effectively to address the security challenges of the twenty-first century.

Thus, Bulgaria faced considerable challenges in defining its defense requirements as a member of NATO, and later of the EU, developing affordable and interoperable capabilities, promoting inter-agency cooperation, and assuring effective and transparent financial, planning, and resource allocation procedures. ${ }^{1}$ At the same time, operations research, modeling, and simulation are an area of active research worldwide with a wide spectrum of applications, including planning, decision support, and conducting exercises. In addition, with the rapid developments in information technology during the past two decades, the methodology of modeling gained an increasingly prominent role in military, economics, social affairs, industry, education and other domains.

* The paper reflects Bulgaria's experience in implementing a capacity-building research project, entitled "Operations Research Support to Force and Operations Planning in the New Security Environment," sponsored by the NATO Science for Peace Program. The authors of this paper are project co-directors. Detailed reports are available at www.gcmarshall.bg/ sfp981149.

1 All these are included among the PAP-DIB objectives. 
The benefits that a decision support institution offers to a nation are many. An institution dedicated to modeling, simulation, and other forms of research related to decision support can provide:

- A single technical focus for decision related matters

- Broad capabilities to address operational problems and shortfalls

- Close and continuing relationships with operational organizations

- Integration of scientific support, planning, and acquisition

- Increased quality of planning and policy development activities

- Reduction of acquisition risk through user-driven prototypes

- Improved integration of prototyping and system implementation

- An improved technical base to support national activities

- Cost-effective utilization of scarce national technical resources.

In NATO as a whole, as well as in several individual NATO nations exists a long tradition of developing and using models and simulations within operations research institutions that are tasked to support the military staffs and administrations with analyses and problem-solving activities. The overall objectives are to save resources or to maximize effectiveness of forces. In the past, developments focused primarily on system-level effectiveness, resource optimization, tactical battle simulations, and logistics.

With the ongoing progress of the process of defense transformation and the appearance of new missions for NATO, new developments are required, in particular the modeling of C4ISR, ${ }^{2}$ asymmetric conflicts, terrorist activities, net-enabled capabilities, as well as interoperation with other security sector organizations.

\section{Project Objectives}

The main objective of the capacity-building research project addressed by this article was the establishment of a scientific capability in the area of operations analysis in Bulgaria, involving young scientists from civilian institutes and universities. As far as possible, this capability should be organizationally identifiable and should generate a corporate identity, a basis of state of the art knowledge and experience, and a valued reputation for providing decision support to national and international administrations.

The most important of the identified requirements was the need to provide decision support to long-term defense planning, force structuring, and security sector transformation; operations planning (accounting for the potential of network-based operations); and acquisition life cycle management. Other requirements addressed the assessment and adaptation of existing methods in decision support-along with the development of new methods and software tools - to the operation of the security sector, with a focus on the simulation of command and control processes within joint mili-

2 Command, Control, Communications, Computers, Intelligence, Surveillance, and Reconnaissance. 
tary/civilian security organizations that are involved in counterterrorist activities and disaster prevention and response. The overall strategy was to adapt existing methods and tools of operations analysis and create novel ones, and then implement them for the purposes of transforming Bulgaria's security sector to deal with the new security challenges of the post-Cold War era in a cost-effective manner.

The expectation was that the results will improve the effectiveness of force planning, equipment and infrastructure planning, as well as operational planning and the training of forces to operate within a well-integrated security sector, providing for a better balance between national capabilities and Bulgaria's contribution to NATO and the European Union. In parallel, the established operations research community was working to improve the basis for transparency, accountability, and democratic control over security decision making, allowing for more effective use of scarce resources.

The application of state of the art methodology within the decision-making process will result in reduced chances of making incorrect decisions, as well as improvements of the effectiveness of the security system and cost savings. In quantitative terms, a careful and very conservative estimate of 5-10 percent savings of the overall security sector budget in Bulgaria (approximately two billion BGN per annum) would result in total cost savings of 100-200 million BGN, or an equivalent increase in security sector effectiveness.

\section{Institution Building}

Since skills in the field demand rigorous academic and scientific training in physics, mathematics, and information science, the necessary specific expertise and capability in operations analysis, modeling, and simulation needed to be developed and recognized by the Bulgarian government as well as by NATO agencies and defense institutions of NATO nations. Three logical steps of the project were defined, each building upon the others.

The initial step was the creation and organization of a Center for Operations Analysis (COA) in the Bulgarian Academy of Science (BAS) and a team within the Defense Staff College (DSC). This included the selection of young scientists, the identification of models for transfer from NATO, the first acquisition of equipment, the creation of management structures, and the training of the scientists in operations analysis theory and practical approaches. The second step built on the initial creation of the COA and DSC with the actual transfer of the selected models, their adaptation and potential upgrades for specific conditions and applications in Bulgaria, the creation of databases and scenarios, and the planning of real applications in analyses and exercises. In parallel, new approaches were required in areas where models were not readily available, in particular for $\mathrm{C} 2$ processes, network-enabled capabilities, and new NATO missions. The third step built on the state of the art methodology developed and/or adapted in the second step and performed actual analyses of problems in the integrated security sector of Bulgaria, the broader region of Southeastern Europe, and within the NATO context, and also provided support for the relevant exercises. All three steps relate directly to the objectives and pre-established criteria of success. In practice, a continual reitera- 
tion of the process was required based on the experience gained. This resulted in a continuous feedback, learning and improving of the methodology and the build-up of high value expertise and continuity.

Due to unforeseen obstacles and ongoing work on other projects, the initial planning and logical construction of the work packages required a continuous adaptation of plans and approaches in order to react to real-life problems. This in itself was a matter of gaining experience in real-world environments, and brought valuable results. As a consequence, the development of the COA and DSC was more of an evolutionary process, instead of being conducted in a top-down manner.

The pre-established criteria of success allowed for the straightforward quantification of results and provided a formula to assess the project's overall performance. The highest value was attached to achievements that helped support decision making in Bulgaria's security sector organizations, thus leading to more efficient use of resources and improved preparedness of Bulgaria's security sector to deal with new security challenges. Some of the criteria were met at 100 percent success (or even more in certain cases, due to the number of project contracts by Bulgarian ministries). Other criteria were not met as fully, because of obstacles and the practical development of the project. In particular, the goal of sending young scientists to NATO agencies was not realistic because of the lack of interest among the young scientists and a lack of relevant vacancies.

\section{Organization}

It is common at the beginning of a project such as this to be confronted with the challenge of how to demonstrate the value of the scientific solutions and at the same time to get the support and funding for developing the required capabilities that will allow those solutions to be achieved. Typically, a sponsor who is convinced of the value of such an institution has to provide the necessary initial funding. In the case of the present project, the NATO Science for Peace Program was this initial sponsoring agent.

In this context, we can consider the project to have been successful if and only if national high-level decision makers and decision bodies take advantage of the opportunity to make use of advanced decision support based on rational scientific methodology and are prepared to continue the funding of an institution for operations analysis. This institution requires an organizational structure, integration into the national decision structure, a link to industry and science bodies, a continuous adaptation of the newest technologies, and a clear corporate identity.

The fundamental principles of such an institution's functioning need to be established based on the experiences of other countries, as well as NATO and the EU. Important issues relate to the following principles:

- The neutrality principle implies a "not-for-profit" institution, with complete independence from commercial interests. This leads to professional integrity and objectivity, and the ability to provide decision makers with unbiased advice. A clear responsibility that sets the institution apart from a for-profit firm is that it seeks successes far beyond commercial accomplishments. 
- The continuity principle implies the development and maintenance of a corporate knowledge base, with long-term employees and a systematically created repertoire of models, databases, and experience. The motivation of employees to stay in the institution is based on salaries and working conditions that are better than in competing industries, on the reputation of the institution, and the desire to serve the country by achieving better solutions to security challenges.

- The flexibility principle implies an organization that is able to respond quickly to decision requirements without bureaucratic obstacles. This required flexibility will not exist if the institution is only able to start working after time-consuming bidding processes in a competing environment, or after the completion of mandatory lowlevel administrative and bureaucratic procedures.

- The scientific state of art principle implies a close link to the scientific and relevant industrial communities within the nation and internationally. Results of the institution's work - in particular its research methodologies and longer-term experiences-will need scientific review at conferences and workshops. The positive elements of scientific (not commercial!) competition will be utilized as much as possible for the support of expertise, maintenance of the knowledge base, and motivation of scientific staff members.

- The customer relationship principle implies that the different cultures of science and decision making (e.g., military, political) require an organizational structure that creates the most effective possible synergies and establishes the requirement of cooperation.

- The management principle implies planning and organizing resources to produce cost-effective and timely products that meet decision requirements. It thus deals with people, materials, money, and equipment, but the key element in the process is people-their motivation, objectives, strengths and weaknesses, social behavior, and group dynamics.

As result of performing some projects and making some evolutionary adjustments due to organizational obstacles, funding constraints, existing responsibilities, and cultural conditions, a structure of two principal core teams in operations analysis is presently in place. This is very likely not the final organizational structure, because several decisions related to the future of operations analysis in Bulgaria are currently pending. The fact that these decisions are being considered can be seen as a consequence of the high-value contributions of the teams to the decision-making process in the administration and the visibility of the group's already high level of professionalism.

The separation of the group into two principal core teams has considerable advantages. The COA within the BAS maintains the freedom and neutrality of scientifically developed projects results and, if it were to receive a basic level of funding and support, could become a not-for-profit institutional resource for several ministries in Bulgaria. In addition, it is able to generate and maintain links to the scientific and industrial communities; the results of its work are reviewed on a peer-to-peer basis; and the scientists working within the institution can be employed under standard civilian con- 
ditions. The team within the DSC, on the other hand, has very close relationships with the Ministry of Defense. As a result, it has a high level of internal knowledge and confidence, which are necessary prerequisites for the team to make appropriate contributions to the decision process. Another advantage of having a close relationship with the defense establishment is that this team has the ability to take on projects that deal with sensitive issues.

For the future, it is important that both units are formally linked by agreements that require them to work together closely and help to create the desired synergies. A general framework agreement between the COA (BAS) and DSC already exists. In addition, we envision a framework agreement to form a "Bulgarian Network for Operational Analysis" at the end of the project; this network would include more institutes within BAS, two universities, the DSC, and the Academy of the Ministry of the Interior.

\section{Infrastructure and Methodology}

In NATO as a whole (as well as within several NATO nations) there exists a long tradition of developing and using models and simulations within OA institutions that are tasked to support the military staffs and administrations with analysis and problem solving. Many of these models are made available directly to member countries, because NATO funded their development; other models are proprietary and owned by industry or by individual nations. In general, and at the beginning of our project in Bulgaria, we planned to use NATO-funded models for the purposes of this project. In addition, we considered nationally-developed models as well. Although the models developed and funded by NATO are freely available, the transfer of these models to the Bulgarian context is not a trivial task, given the organizational, training, and documentation obstacles that must be overcome.

For the utilization and execution of the models, simulations, and mathematical tools, a sufficient computer infrastructure is essential. So-called test-beds or model laboratories were necessary in order to provide the environment for testing new concepts of operation and running the models for research, planning, and decision support. In addition, we can use this setup to create the virtual environment for computer-assisted exercises and to demonstrate the feasibility of certain concepts and approaches. These test-beds are in general client-server networks with several desktop computers and graphical user interfaces, which can be linked and structured in manifold combinations for different projects and applications.

After some initial planning, we developed two test-beds or laboratories: the Joint Training Simulation and Analysis Center (JTSAC) in the COA and the Analytical Support for Transformation Laboratory (ASTRA) in the DSC. The JTSAC provides an environment for analysis and exercises in emergency and crisis management. The JTSAC's mission is to be a test-bed for different agencies in the planning, conduct, and analysis of interagency, international, and civil-military cooperation as well as exercises in the area of civil security. JTSAC maintains capabilities primarily for scenario development and analysis; command, control, communications, and computers systems 
architecture development; joint training and exercises; program management; and assessment.

The ASTRA Lab provides analysis, decision support, and training in the areas of capabilities-based force planning, costing, organizational transformation, gaming in support of senior decision makers, decision support to personnel management, acquisition project management, and security and defense research and technology management. It maintains capacity in crosscutting areas such as methods for decision-making and decision-support systems, knowledge management, and computer network and software support.

The training and certification of the scientists participating in the project was a continuous process during the project. Several training courses in operations analysis, computer assisted exercises, modeling and simulation, and defense planning were planned and executed. External experts and advisors were invited for special sessions. In addition, members of the project team participated in several conferences, workshops, and meetings that contributed to the enhancement of their scientific capabilities. In the end, the best training was achieved by working on real projects - there is no substitute for learning by doing.

\section{Customers and Contributions}

It was anticipated that the results of the project would be documented and used either for the direct decision-making process or as bases for the long-term evolutionary development of expertise that will provide a continuous feed of advice to the administration as well as for the improvement of methodology and approaches to the generation of innovative solutions. In this sense, it was of high importance that we involved the decision-making authorities in the process from the beginning and tried to integrate them into the project work as much as possible. This was required for the creation of confidence and trust in the seriousness and professional attitude of the scientific analysts on the side of the "customer" (the government), and for the development of knowledge and familiarity with the decision process on the side of the scientists.

In addition, both teams needed to build up an international reputation for a high level of professionalism and to become integrated into the network of international operations analysts. This was accomplished by their direct involvement in the activities of the NATO Research and Technology Organization (RTO), participation in international workshops and conferences, and work within international projects (e.g., with the European Union, in Ukraine, in various formats in Southeastern Europe, etc.).

Two international conferences were selected as key measures to indicate the level of acceptance of the scientists' work in the international community; the reception given to the work done by the COA and DSC teams at these conferences showed considerable interest in their work and respect for their contributions. The proceedings of these conferences have been published, and as a result the COA and the DSC are beginning to be noticed by the international community as capable assets within Bulgaria.

Besides several smaller contributions, two applications of these newly established capabilities in COA and DSC created high-visibility results: the TACOM exercise and 
the Bulgarian Defense Requirements Review. The MoSPDA ${ }^{3}$ organized the exercise $\mathrm{TACOM}^{4}$ with support from the European Commission. The aim of the exercise was to improve the response capacity and coordination of civil protection structures, experts, and intervention teams by ensuring compatibility and complementarity in their assistance to a country requesting assistance within the context of the Civil Protection Community Mechanism. The COA was tasked to develop the scenarios, utilize the JTSAC, and to execute the computer-assisted exercise in close alignment with field exercises.

In the second important task, COA and DSC are involved in the ongoing Strategic Defense Review, the new Plan 2015 for the development of the Bulgarian armed forces, and the creation of a new national security concept for Bulgaria. One of the scientific results directly utilized by end-users is the Long-term Capabilities-based Defense Planning Methodology. The Guidance on Actualization of Defense Reform and Modernization Plan directly uses concepts and major portions of the COA results. In particular, this Defense Requirements Review indicates a growing understanding on the part of defense decision makers of the need for and the potential uses of operational analysis, and an increasing awareness of the capacity in this area that exists in Bulgaria.

\section{Summary}

In any nation with high social and technical standards, it is essential to develop and maintain a scientific institution that provides support to national decision making based on state of art methodology and knowledge. If this is not available, the nation will face considerable disadvantages in the international economic arena and problems in guaranteeing the security of its people. We see this project as an initial foray toward the required steps in the establishment of such an institution.

The capacity-building effort for the use of operations analysis in Bulgaria generated many opportunities for improved decision making in the national administration as well as in other nations in Southeastern Europe. Although the developed capability needs further support, the positive results in several projects - in particular TACOM and the Defense Requirements Review - show the high value of the contributions to national decision making.

The main lessons learned from this project are as follows:

- It is a challenge to create a network that links institutes within the BAS to academic institutions within the ministries of defense and the interior

- In the current environment, it is difficult to find, motivate, and keep involved young operations analysts, in particular at the Bulgarian Academy of Sciences

Ministry of State Policy for Disasters and Accidents

4 European Union Terrorist Act Consequences Management in South Eastern Europe 2006 (EU TACOM-SEE 2006). 
- The culture of using operations analysis and computer-assisted exercises requires considerable further promotion and improvement among end users in the security sector

- Support from international experts and the integration of Bulgarian practitioners into international projects is essential for the survival of the nation's operations analysis capability

- The methodology of applied operations analysis, modeling, and simulation should be a key element in academic curricula

- The existence of the established capacity can only be sustained if sufficient funding and tasking by the end users is guaranteed

- The capacity of a "think tank" institution is a high-value asset in national security structures, if it is used effectively

- In comparison to other defense assets, or in relation to potential wrong decisions in catastrophic situations that might occur in the absence of such an institution, the potential payoff is very large relative to the investment required to create such an institution.

Many elements of the present organization are not yet optimal, and need to be adjusted for future challenges, in particular when competing under commercial conditions in international environments. The culture of operations analysis, systems analysis, computer/model assisted exercises, modeling, simulation, or operating in virtual environments needs further development and requires promotion within the national end-user domains. Based on the excellent fundamental education that it provides to its young scientists, Bulgaria should further develop and maintain a competitive establishment for these activities. This will be a security asset of high value that can fill many gaps in the region of Southeastern Europe and the wider Black Sea area, and can eventually make a highly valued contribution to similar institutions in NATO and the European Union. 